\title{
A lidar-radar framework to assess the impact of vertical structure on interferometric coherence
}

\author{
Matthew Brolly, Member, IEEE, Marc Simard, Member, IEEE, \\ Hao Tang, Ralph O Dubayah, Member, IEEE, Justin P Fisk
}

\begin{abstract}
In this paper, we present novel modelling approaches to investigate the sensitivity of radar interferometric coherence to variations in the vertical forest canopy profile. We introduce a common framework applicable to model radar microwave extinction and structure from lidar data. To perform this analysis, we make use of interferometric data from the UAVSAR (Uninhabited Aerial Vehicle Synthetic Aperture Radar) L-band radar and full waveform lidar data from LVIS (Laser Vegetation Imaging Sensor). The data sets were acquired over the Laurentides Wildlife Reserve forest, Quebec, Canada. A two-fold analysis of the framework to estimate interferometric coherence is undertaken. First, a sensitivity analysis is performed by incorporating lidar waveform Legendre descriptions into two adapted independent polarimetric interferometry models. Second, we examine the effectiveness of using lidar data in this novel way to model radar interferometric coherence. Where appropriate, coherence estimates are obtained using Legendre solutions up to $4^{\text {th }}$ order and at resolutions up to $75 \mathrm{~m}$. The maximum $r^{2}$ values between modelled outputs and observed coherence across $\mathrm{hh}$, vv and $h v$ polarisations are shown as $0.51(p<0.05)$ and $0.76(p<0.05)$ at $25 \mathrm{~m}$ and $75 \mathrm{~m}$ pixel resolutions respectively. The introduction of a common framework to combine lidar and radar enables an estimation of the impact of canopy structure on observed interferometric coherence and provides further insight into the feasibility of assuming uniform microwave extinction rates on different scales through forest canopy. The framework's potential lies in its use to assess performance of canopy structure estimates from future spaceborne radar interferometers in synergy with lidar data.
\end{abstract}

Index Terms - Synthetic aperture radar, interferometric coherence, vegetation, forest, lidar, vertical structure, EM extinction.

Paper first submitted XXX. This project was funded by NASA's Terrestrial Ecology program (WBS 281945.02.61.01.69) and through funding for the NASA-ISRO SAR Science Definition Team (NNX12AQ80G). Part of this research was carried out at the Jet Propulsion Laboratory, California Institute of Technology, and was sponsored by the National Aeronautics and Space Administration. Partial funding was also provided by the University of Brighton Rising Stars Scheme.

M. Brolly is with the School of Environment and Technology, University of Brighton, Brighton, UK (e-mail: m.brolly@brighton.ac.uk)

M.Simard, is with the Jet Propulsion Lab, Pasadena, CA USA (e-mail: marc.simard@jpl.nasa.gov).

R.O.Dubayah, H.Tang, are with the Department of Geographical Sciences, University of Maryland, College Park, MD, USA. (e-mail: dubayah@umd.edu, htang@umd.edu).

J.P.Fisk is with Applied Geosolutions, Newmarket, NH, USA (jfisk@appliedgeosolutions.com).

\section{INTRODUCTION}

Tnterferometric Synthetic Aperture Radar (InSAR) using Isingle or multi polarisation modes [1,2] has emerged as a viable alternative to estimate forest metrics necessary to accurately estimate aboveground forest biomass [3, 4].

InSAR has been used extensively to generate Digital Elevation Models (DEM) [5], and more recently, to study forest structure [2, 6-10], in particular, canopy height [1114]. The response of interferometric coherence and phase to forest structure has been shown to be dependent on microwave polarisation [15-17]. This dependence results from the various scattering mechanisms such as multiple, surface, and volume scattering, and enables the location of the ground below the canopy and to infer forest height through application of a 'Random Volume over Ground' (RVoG) model [3, 18]. This model assumes constant microwave extinction through the canopy volume, manifesting as an exponentially diminishing energy level as a function of canopy depth. This modelling approach adopts generic canopies of equally sized objects and provides little direct association with the complex scatterer distribution associated with a natural canopy. Although this model, and the like, produce robust height estimates [19-24] there is potential for improvement through a consideration of this structural complexity. To assess the impact of forest vertical structure on interferometric coherence, a more realistic interpretation of canopy extinction should see a variation with respect to the vertical distribution of material within the forest canopy. The microwave extinction can be a significant parameter in the estimation of canopy height when using a RVoG type model as shown in Fig 1. The effect on height estimation when incorporating a varying extinction is shown to great effect in [25] and serves as a major influence on the work presented here. Achieving a better characterisation of the vertical forest structure and better considering how extinction varies with structure type, is an essential step towards improved estimation of biophysical attributes such as above ground biomass.

In this paper, we use lidar waveforms to produce a more realistic representation of extinction than simply assuming a constant mean value within a particular forest. This representation attempts to account for the apparent complexity of the vertical canopy structure. Full waveform recording lidar such as the Laser Vegetation Imaging System (LVIS) [26] produce detailed vertical backscatter representations of the vertical forest profile and it is these representations that are 
used here to derive forest structural parameters. Synergies between radar and lidar have been investigated in previous studies [9, 27-29] but here, we seek a common modelling framework to link lidar and radar metrics beyond simply the assessment of forest height. We use lidar-derived foliage and woody matter distribution within the canopy [30-35] to predict interferometric coherence and phase values. This is primarily achieved through decomposition of the lidar waveform into a Legendre orthogonal basis commonly used in Polarisation Coherence Tomography (PCT) [36]. We investigate how a canopy height profile representation based on a lidar waveform can facilitate vertically varying canopy structure and microwave extinction as a preliminary sensitivity analysis. This relative sensitivity is then used to predict radar interferometric coherence values obtained from L-Band UAVSAR data at a $25 \mathrm{~m}$ and $75 \mathrm{~m}$ pixel level. Canopy Height Profiles (CHPs) derived from lidar waveforms are used as tools to investigate both the sensitivity of interferometric coherence to vertically varying microwave extinction and the ability to predict radar coherence values through the application of two different lidarradar frameworks. The differences in these frameworks lie in their use of the input vertical profile to model extinction, with one incorporating absolute values associated with the processed waveform and the other taking advantage of relative shape changes. The purpose of this approach, from an applications perspective, is to inform whether radar interferometric coherence, available over large spatial scales, is sensitive to forest vertical structure and to also compare how two different frameworks perform.

In this paper, we present a method to combine knowledge of forest vertical structure obtained from lidar waveforms with interferometric coherence from radar. Vertical structure estimation using SAR is not presented here as a novel idea due to the existence of effective methods for multi baseline acquisitions using coherence tomography [37], PolInSAR [1], and PCT. Here, a single acquisition and single polarisation SAR interferometric baseline is used to present a relationship between vertical canopy distribution, indicated by lidar waveform return intensity, and microwave extinction rate through the canopy. The latter is a parameter essential to effective use of the RVoG model $[3,18]$ and its variants [38]. This method regarding canopy extinction contrasts with that of coherence tomography. Coherence tomography has produced excellent results when recreating volumetric structures and vertical profiles of forest canopies, notably in [39, 40], [41], and [42]. Other work by [43] has provided an alternative approach to determining vertical forest structure using circular SAR with excellent vertical distribution results. The methods presented in this study do not seek to supersede these but provide an alternative and comparable approach, combining the properties of lidar and radar data as well as scattering models.

To avoid confusion, throughout this text, correlation is discussed as the result of statistical comparison between modelled and observed data, generally presented using $\mathrm{r}^{2}$. Coherence is discussed as the output of the radar interferometry process and also for the outputs predicted using the examined models. These outputs then form the comparison.

\section{Methods AND Model DeVelopment}

\section{A. Datasets}

UAVSAR [44] is an airborne SAR system which flies at a nominal altitude of $12.5 \mathrm{~km}$ and operates at L-Band with full quad polarisation capabilities. It is designed to acquire repeat pass interferometric data with a range resolution of $1.8 \mathrm{~m}$. With a typical range swath of $20 \mathrm{~km}$, the look angle varies between $25^{\circ}$ and $65^{\circ}$.

The LVIS sensor [26] is a lidar system developed at NASA's Goddard Space Flight Center with a $20-25 m$ footprint. It is effectively a pulsed laser altimeter that measures range in the vertical direction by timing a short ( $<10 \mathrm{~ns}$ duration) $5 \mathrm{~mJ}$ pulse of laser light $(1064 \mathrm{~nm})$ between the instrument and target surface with repetition frequency of $500 \mathrm{~Hz}$. It has been used extensively in cited forestry studies [32, 45, 46] and is trusted to an extent that it is commonly used for height verification of field studies and other remote sensing surveys [14, 31, 47, 48].

\section{B. Site Description}

Radar data from UAVSAR and lidar data from LVIS were acquired in August 2009 at the Réserve Faunique des Laurentides (Laurentides Wildlife Reserve), situated in the Canadian province of Québec between Québec City and Saguenay $\left(\sim 47.7^{\circ} \mathrm{N},-71.3^{\circ} \mathrm{W}\right)$. The site is spread across an area of $7861 \mathrm{~km}^{2}$ and features an elevational gradient of $1000 \mathrm{~m}$ with a transition from deciduous temperate to boreal coniferous forest. The forest includes mature forest within conservation units, as well as managed stands in forest extraction zones [49]. The nature of the site allows analysis across a broad gradient in forest composition, structure and successional stage [50].

The radar data covers a large proportion of the area approximately bounded by $\left(-71.43^{\circ} \mathrm{W}, 47.72^{\circ} \mathrm{N}\right)$ in the North West and $\left(-71.24^{\circ} \mathrm{W}, 47.47^{\circ} \mathrm{N}\right)$ in the South East. The data is contained within an area measuring approximately $26.1 \mathrm{~km}$ north to south and $14.5 \mathrm{~km}$ east to west. The repeat-pass interferometric acquisitions were performed within approximately 45 minutes with an interferometric baseline of $65 \mathrm{~m}$. The temporal decorrelation associated with interferometry over forested areas was measured to be around 0.1 [49]. Within this area, a much smaller swath of LVIS data is available bounded by the latitudes of the radar acquisition with the extremes of the lidar data located in the North East at $(-71.23 \mathrm{~W}, 47.71 \mathrm{~N})$ and South West at $(-71.29 \mathrm{~W}, 47.55 \mathrm{~N})$. The data runs in a NE to SW direction with a uniform width of approximately $1.4 \mathrm{~km}$ across track. The lidar data partially overlap the radar data.

\section{LVIS Lidar waveforms and canopy delineation processes}

Raw lidar waveforms have been processed to enable their use as proxies for radar canopy extinction and vertical biomass distribution. The basis of the processing is in the removal of background noise, and the delineation of canopy, and ground contributions. To remove the background noise of the data the sigmean noise value associated with the dataset was subtracted from all data values. Additionally a multiplication of 3.75 times the standard deviation of the $1^{\text {st }} 100$ 'noise' returns was used as an ad hoc noise filtering technique to account for the existence of anomalous signals prior to canopy interaction. 
These first 100 returns are assumed to consist only of background noise with zero contribution from the forest. They are considered a reasonable assessment of the Sigmean value embedded in the LVIS data file. Upon noise removal all nonzero values remaining in the dataset can be considered either canopy or ground return. The format of the data then appears cropped at the upper canopy extreme and directly below the ground contribution. The specific ground contributions were identified by analysing the waveform data to detect the occurrence of Gaussian signatures, a method thoroughly described in [51]. It is assumed that the position of each detected Gaussian response within the LVIS waveform can be used to calculate the mean elevation of a specific reflecting surface within the laser footprint. This allows decomposition of the waveform into separate Gaussian components.

\section{Lidar canopy height profiles (CHP)}

Lidar Canopy Height Profiles (CHP) have been generated for this study using techniques outlined in previous high impact studies ([33] ; [34] [31]; [30]; [52]). More complete descriptions and testing of the processes can be found in these cited works. CHPs serve as interpretations of vertical extinction through the canopy by representing the vertical canopy biomass profile, which differ from the raw waveforms as they compensate for the reduced energy transmission with increasing canopy depth. The process involves several steps, beginning with the raw lidar waveform initially followed by the noise removal described in section 2.3. Further to noise removal, the ground and canopy contributions are normalised by the total forest based signal to form the functions $C_{n}(z)$ for canopy and $G_{n}(z)$ for ground. The transmittance height profile (TransHP), a preliminary dataset preceding the CHP calculation, is then calculated as shown in equation (1). In this equation the canopy contribution is normalised by the total wave contribution (canopy + ground). This accounts for the relative level of ground contribution to the total waveform. At any point where the ground contribution is higher the TransHP will be lower, as would be expected from an extinction relationship. Within the denominator of equation (1) the multiplication factor of 2 is necessary to represent the higher soil albedo than the associated canopy ([34], [52], [53]). The level of this albedo has been recorded at more than twice the typical level of forests (10-15\%) with soils shown to produce values up to $40 \%$ [54]. It is worth noting that this factor applied to the ground component could be adapted to suit specific forest conditions as required, possibly accounting for greater reflectivity apparent at different radar frequencies.

$$
\operatorname{TransHP}(\mathrm{z})=\frac{\sum_{z}^{z_{\max }} C_{n}(z)}{\sum C_{n}(z)+\left(2 \sum G_{n}(z)\right)}
$$

This leads to the cCHP (cumulative Canopy Height Profile) calculation using equation (2) and its derivative the CHP equation (3). The cCHP can be calculated by adopting the MacArthur-Horn transform [55] as shown in equation (2), to model occlusion as derived from the Poisson distribution [56].

$$
c C H P(z)=-\ln (1-\operatorname{Trans} H P(z))
$$

Due to the direct measurement of intercepted surfaces made by lidar, it is necessary that this measurement be corrected for attenuation lower in the canopy to allow a profile to be established while assuming equal energy interception at each canopy level. The cCHP (equation (2)) follows the occlusion transformation of the transmittance profile (equation (1)) and is used to find the derivative profile, the CHP (equation (3))[31].

$$
C H P(z)=\frac{d(c C H P(z))}{d z}
$$

The CHP can be viewed as the apparent description of the vertical distribution of woody and foliar biomass within the vertical profile of the forest. This lidar forest metric is regularly used in the literature to model biomass distribution but lacks a significant contribution from the stem layer. The absence of this significant contributor to the biomass profile is in keeping with the nature of optical nadir looking systems. Consequently it is accepted as a study limitation that it may not accurately represent the relative significance of biomass detected at off nadir look angles at which SAR operates. To reduce the potential impact of this limitation, reference should be made to studies such as [57] which indicate that at least for "typically" rough and non-flooded forest floors, scattering contributions from stem-ground interactions associated with L-Band offnadir data collection are not of high importance. As such this issue lies beyond the scope of this study. Although this portrayal of the vertical structure is limited, it does increase upon the realism portrayed by typical forest radar scattering models offered by the Water Cloud [58], and RVoG models. Both cited models predict extinction behaviour through the canopy using a constant medium specific extinction coefficient assuming vertical homogeneity of biomass. This method regularly produces robust results but ignores structural complexity, the significance of which is explored in this study. Examples of the shapes of these CHP waveform descriptions are shown in Fig 2. A constant mean extinction similar to that used in the above mentioned models would be represented by the $0^{\text {th }}$ order, as indicated by P0(x) in Fig.3.

\section{E. Legendre Polynomials}

To describe the CHPs mathematically and allow their use as inputs into interferometric coherence models, we use Legendre polynomials. These have been extensively used in Polarimetric Interferometric Synthetic Aperture Radar (PolInSAR) [36] [59]; [60].

The Legendre series is described using equation (4) with 5 polynomial orders visualised in Fig 3.

$$
L(x)=\sum \mathrm{a}_{n} P_{n}(x)
$$


Where $a_{n}$ is the Legendre coefficient, equation (5), and $P_{n}(x)$ represents the Legendre polynomials, equation (6), as a function of $\mathrm{x}$; which for this study is a function of forest height.

$$
\begin{aligned}
& \mathrm{a}_{n}=\frac{2 n+1}{2} \int_{-1} L(x) P_{n}(x) \cdot d x \\
& 0^{\text {th }} \text { order }=\mathrm{P}_{0}(\mathrm{x})=1 \\
& 1^{\text {st }} \text { order }=\mathrm{P}_{1}(\mathrm{x})=\mathrm{x} \\
& 2^{\text {nd }} \text { order }=\mathrm{P}_{2}(\mathrm{x})=(1 / 2)\left(3 \mathrm{x}^{2}-1\right) \\
& 3^{\text {rd }} \text { order }=\mathrm{P}_{3}(\mathrm{x})=(1 / 2)\left(5 \mathrm{x}^{3}-3 \mathrm{x}\right) \\
& 4^{\text {th }} \text { order }=\mathrm{P}_{4}(\mathrm{x})=(1 / 8)\left(35 \mathrm{x}^{4}-30 \mathrm{x}^{2}+3\right)
\end{aligned}
$$

This mathematical representation was found to be accurate in all cases, even with low polynomial orders and for waveforms exhibiting multiple peaks.

We acknowledge the relevance of this approach to existing single baseline interferometry data and the restrictions imposed on the use of multiple Legendre polynomial orders [36]. But here we are able to extend to higher orders through use of independent waveform descriptions. Most importantly, the Legendre polynomials enable a common framework between lidar waveforms and interferometric coherence which will be examined using two interferometric coherence models: the Garestier \& Le Toan Model [25] and the Cloude Model [36] .

\section{F. Interferometric Coherence Models}

We present two separate and independent models of interferometric coherence to assimilate lidar-derived CHPs. The two models of interest are taken from Garestier \& Le Toan [25] and Cloude [36] and are adapted for use with lidar waveforms (equations (9) and (12) respectively). The use of two different models in this study is justified due to their existing differences. The major difference between the models is how they account for the vertical profile. In the Garestier \& Le Toan model (G\&LT) the coherence estimation is possible using the vertical profile of return intensity as a direct proxy for extinction. In the Cloude [36] model the profile shape and its relative variations are the driver. In both cases a vertical and incremental consideration of interferometric coherence is possible, allowing for critical analysis of coherence variations as a consequence of variable extinction.

\section{1) Garestier \& Le Toan (G\&LT) Model}

In Equation (7) (taken from [25]), the simple case of a constant extinction within a random volume was modelled. However, the extinction $\sigma_{\mathrm{z}}$ can be replaced with a Legendre polynomial expansion related to the CHP of a forest pixel.

$$
\begin{aligned}
& \gamma_{v}\left(h_{v}, \sigma_{z}\right)=\frac{\int_{0}^{h_{v}} e^{\frac{2 \sigma_{z} z}{\cos \theta}+j k_{z} z} d z}{\int_{0}^{h_{v}} e^{\frac{2 \sigma_{z} z}{\cos \theta}} d z} \\
& \gamma_{v}\left(h_{v}, \sigma_{z}\right)=\frac{1}{1+\frac{j k_{z} \cos \theta}{2 \sigma_{z}}} \cdot \frac{e^{\left(\frac{2 \sigma_{z} h_{v}}{\cos \theta}+j k_{z}\right) h_{v}}-1}{e^{\frac{2 \sigma_{z} h_{v}}{\cos \theta}}-1}
\end{aligned}
$$

If the extinction increases linearly with depth into the canopy (z) such that $\sigma_{\mathrm{z}}(\mathrm{z})=\alpha \mathrm{z}$, Equation 7 can be rewritten as follows:

$$
\gamma_{v}\left(h_{v}, \alpha\right)=\frac{\int_{0}^{h_{v}} e^{-\frac{2 \alpha z^{2}}{\cos \theta}+j k_{z}\left(h_{v}-z\right)} d z}{\int_{0}^{h_{v}} e^{-\frac{2 \alpha z^{2}}{\cos \theta}} d z} .
$$

The extinction used here represents a typical $1^{\text {st }}$ order Legendre solution, see Fig.3. Integrating with respect to height, the interferometric coherence resulting from a linearly varying extinction can then be calculated at the chosen height increments $\mathrm{dz}$.

$$
\gamma_{v}\left(h_{v}, \alpha\right)=e^{-\frac{\cos \theta \ell_{z}^{2}}{8 \alpha}+j k_{z} h_{v}} \frac{\operatorname{erf}\left(\frac{j \cos \theta k_{z}+4 \alpha h_{v}}{2 \sqrt{\frac{2 \alpha}{\cos \theta}} h_{v}}\right)-\operatorname{erf}\left(\frac{j k_{z}}{2} \sqrt{\frac{\cos \theta}{2 \alpha}}\right)}{\operatorname{erf}\left(\sqrt{\frac{2 \alpha}{\cos \theta}} h_{v}\right)}
$$

As shown in Garestier \& Le Toan [25], when different values of $\alpha$ are used, the coherence and phase for each height will be different. Similarly, the existence of an extinction value greater than zero at the top of the canopy will offset the coherence and phase of the volume.

The Legendre approximations of extinction (CHPs) can then be applied to this model. The level of extinction and offset is directly determined from the waveform and associated polynomials. Moving to higher Legendre orders is possible using the $\alpha$ function to provide more complex mathematical descriptions of vertical variations.

Some examples of Legendre solutions are shown in Fig. 2 along with the representative CHP. The forest height is derived from the LVIS waveform. The associated mathematical functions of these curves are included in the interferometric coherence calculation using the exponent term $\alpha$ of equation (8). In the formulation of equation (8) the extinction at the top layer is considered null with extinction increasing linearly with depth. For the examples that do not consider a constant extinction coefficient there is an increased likelihood that the extinction value at the top layer will be offset from zero due to the independent fitting of the Legendre polynomials. The G\&LT model uses the direct values taken from the CHPs to model extinction and height specific coherence estimations.

\section{2) Cloude Model}

In Cloude [36] it was shown how polarised SAR interferograms can be combined to estimate structural parameters of vegetation, and not restricted by underlying ground topography or apparent vegetation height. Using estimates of vegetation height and ground phase, the vertical structure function of a forest could be reconstructed through removal of the topography phase term and by normalising an integral through a change in variable. These steps allowed for a structural function to be defined using a Legendre series starting from the basic definition of coherence defined in equation (10). In the Cloude scenario, $z$ represents the height 
from the ground contrary to its use in the G\&LT model where it represents depth.

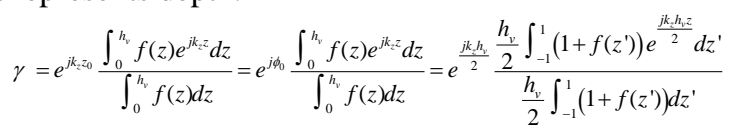

The Legendre series definition (equation (4)) can be factored in to equation (11) and simplified to the form of equation (12).

$$
\begin{aligned}
& \gamma=e^{\frac{j k, h_{k}}{2}} \frac{\int_{-1}^{1}\left(1+f\left(z^{\prime}\right)\right) e^{\frac{j k_{k}, h_{2}, z}{2}} d z^{\prime}}{\int_{-1}^{1}\left(1+f\left(z^{\prime}\right)\right) d z^{\prime}}=e^{j k_{k}} \frac{\int_{-1}^{1}\left(1+\sum_{n} a_{n} P_{n}\left(z^{\prime}\right)\right) e^{j k_{z} z} d z^{\prime}}{\int_{-1}^{1}\left(1+\sum_{n} a_{n} P_{n}\left(z^{\prime}\right)\right) d z^{\prime}} \text { where } k_{v}=\frac{k_{z} h_{v}}{2} \\
& \gamma=e^{j k_{v}} \frac{\left(1+a_{0}\right) f_{0}+a_{1} f_{1}+a_{2} f_{2}+\ldots . a_{n} f_{n}}{\left(1+a_{0}\right)}
\end{aligned}
$$

The explicit forms of the coherence basis functions $\left(f_{n}\right)$ of equation (12), up to the $4^{\text {th }}$ order, are shown in equation (13) [36]. From equation (12) it is then possible to define coherence using the Legendre coefficients, lidar defined volume height, coherence basis functions, and through initial estimation of the vertical wavenumber $\left(\mathrm{k}_{\mathrm{z}}\right)$ using characteristics of the relevant SAR acquisition. For datasets used in this study the vertical wavenumber is defined by the average characteristics of the UAVSAR acquisition, not accounting for variable baselines. Variable baselines and varying incidence angles are not studied here and provide scope for further work. This decision was taken due to the small variations evident in the overlapping dataset. Inverse to this process, if coherence is known, such as that observed from UAVSAR, the vertical structure functions can also be estimated.

$$
\begin{aligned}
& f_{0}=\frac{\sin k_{v}}{k_{v}} \\
& f_{1}=i\left(\frac{\sin k_{v}}{k_{v}^{2}}-\frac{\cos k_{v}}{k_{v}}\right) \\
& f_{2}=\frac{3 \cos k_{v}}{k_{v}^{2}}-\left(\frac{6-3 k_{v}^{2}}{2 k_{v}^{3}}+\frac{1}{2 k_{v}}\right) \sin k_{v} \\
& \left.f_{3}=i\left(\frac{30-5 k_{v}^{2}}{2 k_{v}^{3}}+\frac{3}{2 k_{v}}\right) \cos k_{v}-\left(\frac{30-15 k_{v}^{2}}{2 k_{v}^{4}}+\frac{3}{2 k_{v}^{2}}\right) \sin k_{v}\right) \\
& f_{4}=\left(\frac{35\left(k_{v}^{2}-6\right)}{2 k_{v}^{4}}-\frac{15}{2 k_{v}^{2}}\right) \cos k_{v}+\left(\frac{35\left(k_{v}^{4}-12 k_{v}^{2}+24\right)}{8 k_{v}^{5}}+\frac{30\left(2-k_{v}^{2}\right)}{8 k_{v}^{3}}+\frac{3}{8 k_{v}}\right) \sin k_{v}
\end{aligned}
$$

The process outlined in Cloude [36] can be used to obtain relative vertical profiles of the canopy through inversion of coherence data. In this study the inverse of this process is performed. Coherence is here estimated using height and vertical structure measurements derived from lidar waveforms. When applying the inverse process of curve estimation, using the coherence data, the Cloude model is limited by the number of baselines and the subsequent data availability. However, these restrictions are not an issue in the forward modelling case. We use the estimated curves up to a maximum of $4^{\text {th }}$ order, due to the limited improvement in accuracy offered by higher Legendre orders. The Cloude model uses the shape of the profile as an indicator of coherence. This characteristic effectively creates a strong relationship in the Cloude model between coherence and profile height. With reference to Cloude [36] this is a feature of the model, included to minimise height estimation errors in the presence of extinctions ranging from 0 to $1 \mathrm{~dB} / \mathrm{m}$. The Cloude model inherently constrains the range of extinction levels proposed from the relative structure function in order to maintain a range of acceptable values.

In summary the G\&LT and Cloude models incorporate the vertical profiles as extinction inputs and as biomass profile shapes respectively. The work flow to provide the modelled coherence values and then perform the comparison with empirical data using both models is shown in Fig. 4.

\section{RESULtS}

To highlight the accuracy of matching waveforms with Legendre polynomials Table 1 is included. The higher correlations associated with higher polynomial orders is immediately apparent.

\section{A. Modelled coherence sensitivity to variable vertical extinction}

The coherence sensitivity to the vertical extinction profile is examined using the two radar coherence models: G\&LT and Cloude. Firstly to establish the sensitivity of coherence to vertical variations of extinction, and secondly to establish if the two coherence models produce realistic coherence estimates. As an example, Figure 5 shows data for two distinct waveforms with significantly different canopy heights with coherence values generated using the G\&LT model for $0^{\text {th }}, 2^{\text {nd }}$ and $4^{\text {th }}$ Legendre orders. The influence of height on coherence is immediately apparent.

Although interferometric coherence is generally accepted to be mainly influenced by volume height, the vertical profile and therefore extinction, also exert significant influence (Figure 5). In a simple preliminary sensitivity test to compare the two approaches, coherence is calculated using the G\&LT and Cloude models independently, assuming a standard forest height of $20 \mathrm{~m}$ consistent across the site. This sensitivity study incorporates only waveforms shown to possess a forest height of $20 \mathrm{~m}$ or greater with coherence values estimated at this fixed 20m height level. Referring to Figure 5 this sensitivity analysis would include waveform \#860297 but not \#860275 due to minimum canopy height requirements, but the scope for structural variation is depicted by the waveforms. Using waveforms that fit the $20 \mathrm{~m}$ criteria (32000 waveforms) ensures a variety of extinction profiles will be available for testing sensitivity to vertical structure.

Of the two models investigated the G\&LT model offers the greater sensitivity to extinction variation at each polynomial order, exhibited by the standard deviation values. The variation increases with CHP Legendre order highlighting greater structural variation sensitivity. As an example, using the $2^{\text {nd }}$ order Legendre descriptions of CHPs, available according to the sensitivity criteria, and measuring at the $20 \mathrm{~m}$ height level, the estimated mean coherence across the dataset is given as 0.88 for the G\&LT model and 0.16 for the Cloude model. Across the dataset the standard deviations are respectively $~ 9 \%$ 
and $<1 \%$ of the mean. This highlights a very obvious difference between the two models when dealing with higher order polynomials. This behaviour is exhibited in the example waveform shown in Fig.6.. For the G\&LT model the difference in height corresponding to one standard deviation of coherence is approximately $0.8 \mathrm{~m}$ at both $2^{\text {nd }}$ and $0^{\text {th }}$ Legendre orders. Using the Cloude model this corresponds to height variations of $\sim 0.1 \mathrm{~m}$ or less at each order. Again the reader is referred to Fig. 6. The larger variation in coherence estimates, associated with different polynomials is shown when using the G\&LT model. This suggests a greater sensitivity to structural profile variations related to the waveform and therefore CHP-derived extinction. This is a consequence of the Cloude model's use of relative profile shape and inherent constraints existing on potential extinction values rather than the G\&LT model's use of the absolute.

The use of relative shape reduces the need for, and the influence of, highly accurate extinction values on the estimated coherence. The differences exhibited also indicate a requirement for a modification to the G\&LT model to allow specific frequency use, and to constrain the range of potential extinction variations within a medium to conform with expectations. This modification also allows direct comparison with the Cloude model output. An example of the comparison following the modification is shown in Fig. 6 for the example waveform. From the data it is evident that the introduction of a multiplicative factor reduces sensitivity in the Cloude model but increases realism in the G\&LT.

\section{1) Multiplicative Factor}

As mentioned in the previous section, a multiplicative factor can be applied to the extinction coefficients represented by the CHPs to better represent L-Band canopy extinction values. Although these values are not reported in terms of vertical variation they allow a relationship to be established with the $0^{\text {th }}$ order Legendre polynomials acting as the mean constant extinction through the profile. Application of this multiplicative factor increases the model's applicability to multiple radar frequencies. It is worth noting here that reducing the incident frequency reduces the impact of a varying extinction on coherence. The multiplicative factor is applied according to equation (14) and can be applied to both the G\&LT and Cloude models but with differing significance.

$$
M F=\sigma_{F d}(d B / m) / \overline{\sigma_{0 t h}}(d B / m)
$$

MF represents the Multiplicative Factor, $\sigma_{F d}$ the frequency dependent extinction coefficient, and $\overline{\sigma_{0 t h}}$ the mean extinction of the $0^{\text {th }}$ order Legendre coefficient.

As the G\&LT model is sensitive to the absolute numerical values of extinction provided by the CHPs it is important to focus on sensitivity to empirically recorded values. The multiplicative factor can be applied to the CHP data to create this product. For this study an average value of $0.15 \mathrm{~dB} / \mathrm{m}$ is identified as an estimate of L-Band extinction [61]. To obtain a mean CHP extinction value corresponding to L-Band levels, the $0^{\text {th }}$ order values (representing a mean constant extinction through the canopy) are collated for all waveforms. The multiplicative factor of equation (14) is then applied with the resultant distribution shown in Fig 7 to give an extinction range 0 to $\sim 0.70 \mathrm{~dB} / \mathrm{m}$. The distribution of extinction values is representative of all waveforms of all heights within the dataset that feature a vegetation contribution.

The inclusion of the multiplicative factor maintains a level of variation exhibited throughout the profile but the dynamic range of extinction is reduced, as is sensitivity to smaller scattering targets. This level of sensitivity is further reduced when examining lower frequencies.

For the Cloude model the inclusion of similar multiplicative factors reduces the observed coherence variation seen for any particular height by subduing the extremes of the profile shape, essentially repeating the process. The effect can be seen in Fig.6 where variation between coherence estimates from polynomials becomes negligible. As a result of this the multiplicative factor is not required for use with the Cloude model. For the G\&LT model a similar reduction in sensitivity to the profile variations is not seen when applying the multiplicative factor. As such what is shown is that mean coherence values are brought in line with those recorded for the Cloude model and most importantly empirically determined SAR extinction values.

\section{2) Sensitivity Summary}

The results regarding sensitivity, described above, suggest that changes in the vertical extinction profile results in coherence variation. This is particularly true when using the G\&LT model, which, unlike the Cloude model, is not as rigidly constrained by canopy height. This feature of the Cloude model ensures that less variation is seen for a forest if the Legendre representations are similar in shape. The fact remains that variation, however small, influences coherence estimated by the models, albeit in different ways and to different levels of significance.

Fig. 6 includes the coherence data comparison produced by the two models using a single waveform example. The data is shown with respect to height within the canopy volume. Although coherence is generally similar, they begin to diverge at greater height as a consequence of varying extinction. As an example we compare the $0^{\text {th }}$ order curves, constant extinction, for both models. The differences existing between the two models are associated with the use of the absolute extinction value by the G\&LT model and the relative profile shape used by the Cloude model. At $0^{\text {th }}$ order the same constant extinction value is used for both models with the G\&LT matching the coherence values associated with the RVoG model whereas the Cloude model output highlights the effect of a constraining feature discussed in Cloude [36]. If the shapes of the CHPs at each order are similar, the Cloude model will produce similar coherence. Where the profile shapes are different, the coherence changes accordingly.

This sensitivity analysis highlights the implications of considering vertical complexity using higher order Legendre descriptions when estimating coherence. If the extinction profile derived from lidar data is similar to that experienced by L-Band radar, it is at these higher levels of complexity that the greatest statistical correlation in coherence between SAR data and lidar models is expected. In the next section, we evaluate 
and compare the modelled coherence using available UAVSAR data. Note that all waveform variations of height and shape, featuring a vegetation contribution, are considered in the remainder of the study with height restrictions no longer in place.

\section{B. UAVSAR Interferometric Coherence Comparison with Lidar Derived Data}

Interferometric coherence values observed from L-Band SAR data using hh, vv, and hv polarisations are compared with modelled coherence values estimated using the lidar derived CHP inputs. All data are originally considered at a pixel resolution of $25 \mathrm{~m}$. The statistics of the comparison are presented in terms of each polarisation, using the Legendre descriptions of the lidar data from the $0^{\text {th }}$ up to a maximum of $4^{\text {rd }}$ order. The major difference associated with increasing Legendre orders is that the higher order CHP representations model the reduction in waveform intensity associated with the area between the canopy and ground responses. Sample data are also discussed at $75 \mathrm{~m}$ pixel level to emphasise greater correlation between modelled and observed data at coarser spatial resolutions.

Figure 8 displays the layout of the test site in terms of the LBand UAVSAR hv coherence data, the lidar derived coherence data using the G\&LT model and the difference between UAVSAR observed minus G\&LT modelled coherence.. The difference image shows areas with significant differences which may correspond to areas of natural (eg. topography, geology) or anthropogenic features such as roads, and clear cuttings.

Table 2 shows the $r^{2}$ correlation between the observed and modelled coherence values. We excluded objects from the analysis that included those classified as water or ground, objects outside a $30^{\circ}$ range of slope angles $\left(0-30^{\circ}\right)$, and those declared anomalous due to missing data or incorrect recording of coordinates. The $r^{2}$ values are significantly improved when setting thresholds to compensate for topographical conditions. These topographical variations distort the identified ground response in the waveform and hence make its detection, and establishment of the local canopy height, difficult. Further work is needed to account for topographical features. Comparison of the UAVSAR observed coherence with the Cloude and G\&LT models only show very slight increase in correlation using higher order polynomials.

The result of averaging the coherence data over a $75 \mathrm{~m} \times 75 \mathrm{~m}$ pixel window significantly improves the $r^{2}$ but no significant improvement is observed at higher polynomial orders.

As a further comparison, Fig 9 highlights the $r^{2}$ values associated with the use of a single mean constant extinction level applied across the entire dataset (i.e. the waveforms being solely used to estimate canopy height). Some indicator extinctions are also included in Table 2 for comparison. In Table 2 the similarities between the Cloude and G\&LT coherence values (using extinction values derived from the waveforms) and those using an independently assigned constant mean extinction, in the manner of the RVoG model (Fig 9), are most apparent in the region of extinction between 0 and $0.6 \mathrm{~dB} / \mathrm{m}$ after which point the $\mathrm{r}^{2}$ values drop off rapidly to 0.21 at $1 \mathrm{~dB} / \mathrm{m}$. Such low values are not evident using the other modelling processes. The $r^{2}$ for the independently defined constant mean extinctions are shown not to exceed the maximum values set by the Cloude and G\&LT models at the $25 \mathrm{~m}$ pixel level. The coherence modelling approach provides a means to assess the impact of the canopy's vertical profile on interferometric coherence which is found to be insignificant. We therefore conclude that the assumption of a constant mean microwave extinction through the canopy is a valid approach at L-band.

\section{1) Garestier \& Le Toan Method}

Fig. 10 shows sample data associated with the $3^{\text {rd }}$ order Legendre dataset following slope filtering at $25 \mathrm{~m}$ resolution. It shows the distribution of radar measured and lidar derived coherence estimates shown with respect to the 1:1 ratio for all examined waveforms. The multiplicative factor and local topographical slope filtering have an obvious effect on the correlation while the remaining bias displayed in the figure may be partially explained by the effects of repeat-pass temporal decorrelation $(\sim 0.1,[49])$. The highest $r^{2}$ is found for hv polarisation using displayed example (although by only 0.03 above the $0^{\text {th }}$ order case) with an $r^{2}$ of 0.51 at $25 \mathrm{~m}$ resolution and 0.74 at $75 \mathrm{~m}$ resolution.

\section{2) Cloude Method}

For the Cloude method the highest $r^{2}$ is found for hv polarisation using the $4^{\text {th }}$ order description (although by only 0.03 above the $0^{\text {th }}$ order case) with an $\mathrm{r}^{2}$ of 0.49 at $25 \mathrm{~m}$ resolution and 0.75 at $75 \mathrm{~m}$ resolution. This data is shown Fig. 10 at $25 \mathrm{~m}$ resolution following slope filtering. The data presents increased correlation associated with removal of areas of significant slope. Similar improvements are evident with the G\&LT model. Both modelling procedures, to a certain extent, validate the assumption of a single constant extinction through the canopy due to the negligible increases in correlation offered by higher orders and hence negligible association with more complex descriptions of vertical profiles. This assumption is validated through the data in Table 2.

\section{DISCUSSION}

This study has presented a novel framework for estimating radar interferometric coherence through direct association with modelled extinction properties obtained from full waveform lidar data. The approach chosen was intended to take advantage of the increased accuracy in vertical structural estimation offered by full waveform lidar to understand the radar observed coherence. This lidar-radar framework ultimately could aid the development of extinction lookup tables from coherence measurements and allow inversion of coherence to infer vertical profile. The potential for a variable extinction/profile look-up table derived from an association of lidar waveforms with interferometric coherence would offer an alternative for determining forest structure from multiple baselines and polarisations. These may offer potential to upscale to large areas for vertical profile estimation where only single baseline SAR data, and minimal lidar training data is available.

In this study it is shown that the correlation between SAR and lidar inferred interferometric coherence is best presented when excluding local topographic slope angles exceeding 30 
degrees and using a coarse spatial resolution of $75 \mathrm{~m}$. The filtering of a particular range of local inclination angles reduces the impact of slope on waveform processing, which is known to increase data variance, and lower the signal amplitude of the ground Gaussian fit. Slope is also responsible for blurring the ground and canopy energy separation in a waveform signal [62, 63 ] hence introducing error. For the finer resolution of $25 \mathrm{~m}$ the Cloude and G\&LT models produced similar results with $r^{2}$ correlation around 0.5 for hv polarisation. However, these values are dwarfed by the $75 \mathrm{~m}$ correlation data, with $\mathrm{r}^{2}$ values increasing to 0.75 and 0.74 respectively. Contrary to initial expectations the improvement offered by increasing vertical complexity beyond the standard constant mean extinction was shown to be minimal. In effect this validates the use of the $0^{\text {th }}$ order Legendre representation of a constant mean extinction individual to each waveform.

A distinct difference between coherence estimates made by the two interferometric models is the severe systematic bias displayed by the G\&LT model in the absence of a multiplicative factor. This bias is not seen in the Cloude model data. The bias is a result of the direct use of waveform derived extinction values that require adjustment for specific frequency compatibility through the introduction of a multiplicative factor. The use of the multiplicative factor may allow comparison with multi-frequency data using values obtained with respect to both the lidar waveform data and reported empirical values. However, this factor reduces the uniqueness and dynamic range of the examined profiles.

The effect of bias on the exhibited interferometric coherence estimates, particularly for the G\&LT model, may result from a combination of several factors. One potential factor is temporal decorrelation due to motion of scatterers between UAVSAR's repeat-passes ( $\sim 45$ minutes). Temporal decorrelation has been shown to decrease observed interferometric coherence by around 0.1 over the course of 45 minute repeat-pass data from UAVSAR [49]. A coherence correction of 0.1 in each instance would improve the proximity of the data to the 1:1 line in both model representations and likely increase statistical similarities with recorded SAR coherence. A second factor is the use of waveforms to establish forest height and define the contribution of the ground component in the coherence process. The apparent height associated with coherence measurements from SAR data will be a function of several factors including the ground contribution at these frequencies. The ground is taken into account when calculating the CHPs and ensures there is never $100 \%$ canopy attenuation in the presence of a ground contribution (equation (1)). In the lidar coherence estimations it is possible that this ground consideration presents an overestimation in the total percentage of energy lost. Assuming the ground contribution should be increased by a factor of 2 may be incorrect at L-Band (soil levels reported in the literature are as high as 40\% [54]. Underestimating the ground contribution causes overestimation of extinction and thus introduces a bias. The third factor is that the models may incorrectly depict the effects of forest height and extinction on radar interferometric coherence, therefore providing coherence estimates above empirical values. In addition, the lidar-derived estimates of L-band extinction may have inaccuracies, particularly because of the vastly different viewing geometries of the side looking radar and nadir looking lidar. The vertical nature of the stems with respect to the nadir looking of the lidar system reduces the immediate comparability of waveform profiles with SAR data. Attempts to correct the CHPs to account for the prominence of stems at non zero incidence angles, by introducing a stem taper function in proportion to the diameter of the trunk at $0.1 \mathrm{~m}$ intervals, was not shown to improve the data sufficiently to warrant further consideration. A final factor is the use of a near field incidence angle rather than the centre swath value. This may have a small effect on the data output considering the location of the LVIS data within the radar scene. The framework presented in this study would be developed greatly through future innovation in waveform processing, serving to address these possible limitations.

\section{CONCLUSIONS}

Given the ability to facilitate mathematical representation within coherence models and the capability to capture structural differences in CHPs, we are confident that the Legendre decomposition approach provides a promising basis for a framework common to radar and lidar modelling. The level of complexity required in the vertical profile extinction description beyond the use of constant mean extinction remains debatable. In this study we have principally asked three questions (i) Can lidar-derived vertical canopy height profiles be used to model vertical variation of microwave extinction? (ii) Can existing SAR interferometric coherence models be used as a common framework for radar and lidar? And (iii) how accurately can lidar waveform data be used to predict radar coherence using this framework, and does this approach warrant use of complex extinction profiles rather than uniform site specific extinction? We have answered these questions through modelled analysis of coherence sensitivity to extinction variation and direct comparison of lidar-derived coherence with L-Band SAR data acquired from Quebec, Canada.

The purpose of such questioning from an applications perspective has been to inform whether radar interferometric coherence, available over large spatial scales, is sensitive to forest vertical structure. The relevance of such a study is rooted in the ecosystem monitoring requirements of next generation SAR sensors such as ALOS-2 PALSAR [64], Sentinel 1 [65], Biomass [66] and NISAR [67]. This need is furthered by the current absence of spaceborne waveform lidar. Relevant properties potentially available from successfully relating radar and lidar forest data include extinction rate, vertical biomass distribution, and height. Each of these can be related in some form to lidar waveform data available with excellent vertical resolution and remain difficult to establish using SAR data alone.

To establish a common radar-lidar framework, two methods of linking lidar waveform data to radar interferometry coherence have been presented. The first uses the model of interferometric coherence variability with canopy depth presented by Garestier \& Le Toan [25], with the lidar waveforms used to provide estimates of extinction variability. The second uses the model of Cloude [36] to estimate coherence from the vertical structure function, producing coherence values based on relative profile shape. The 
coherence sensitivity of both models' data outputs was initially analysed with respect to the CHPs and the use of an increasing complexity of Legendre descriptions. Modelled coherence values were shown to be influenced by profile variation and compared with observed coherence.

The results show potential for future investigations to establish a more robust common lidar-radar framework, one which embraces the importance of determining extinction as an important factor in height estimation. Applying such a framework with the required accuracy for operational use will require further novel thinking and development regarding waveform processing. Overcoming the sensor variations in incidence angle, wavelength and footprint size may also require greater consideration.

\section{REFERENCES}

1] S. R. Cloude and K. P. Papathanassiou, "Polarimetric SAR interferometry," Geoscience and Remote Sensing, IEEE Transactions on, vol. 36, pp. 1551-1565, 1998.

[2] H. Balzter, "Forest mapping and monitoring with interferometric synthetic aperture radar (InSAR)," Progress in Physical Geography, vol. 25, pp. 159-177, 2001.

[3] K. P. Papathanassion, S. R. Cloude, A. Reiber, and W. M. Boerner, "Multi-baseline polarimetric SAR interferometry for vegetation parameters estimation," Geoscience and Remote Sensing Symposium, 2000. Proceedings. IGARSS 2000. IEEE 2000 International, vol. 6, 2000.

[4] K. P. Papathanassiou and S. R. Cloude, "Single-baseline polarimetric SAR interferometry," Geoscience and Remote Sensing, IEEE Transactions on, vol. 39, pp. 2352-2363, 2001

[5] N. Baghdadi, S. Cavelier, J. P. Chiles, B. Bourgine, T. Toutin, C. King, et al., "Merging of airborne elevation data and Radarsat data to develop a Digital Elevation Model," International Journal of Remote Sensing, vol. 26, pp. 141-166, 2005

[6] J. Lee, M. Grunes, T. Ainsworth, I. Hajnsek, T. Mette, and K. Papathanassiou, "Forest classification based on L-band polarimetric and interferometric SAR data," in ESA Special Publication, 2005, p. 6.

[7] R. N. Treuhaft and P. R. Siqueira, "The calculated performance of forest structure and biomass estimates from interferometric radar," Waves in Random Media, vol. 14, pp. 345-358, 2004.

[8] S. R. Cloude and K. P. Papathanassiou, "Three-stage inversion process for polarimetric SAR interferometry," Radar, Sonar and Navigation, IEE Proceedings-, vol. 150, pp. 125-134, 2003.

[9] P. Hyde, R. Dubayah, W. Walker, J. B. Blair, M. Hofton, and C. Hunsaker, "Mapping forest structure for wildlife habitat analysis using multi-sensor (LiDAR, SAR/InSAR, ETM+, Quickbird) synergy," Remote Sensing of Environment, vol. 102, pp. 63-73, 2006.

[10] J. Askne and M. Santoro, "Selection of forest stands for stem volume retrieval from stable ERS tandem InSAR observations," Geoscience and Remote Sensing Letters, IEEE, vol. 4, pp. 46-50, 2007.

[11] M. Simard, K. Zhang, V. H. Rivera-Monroy, M. S. Ross, P. L. Ruiz, E. Castañeda-Moya, et al., "Mapping height and biomass of mangrove forests in Everglades National Park with SRTM elevation data," Photogrammetric Engineering and Remote Sensing, vol. 72, pp. 299311, 2006.

[12] S. Solberg, R. Astrup, O. M. Bollandsås, E. Næsset, and D. J. Weydahl, "Deriving forest monitoring variables from X-band InSAR SRTM height," Canadian Journal of Remote Sensing, vol. 36, pp. 68-79, 2010.

[13] M. Isola and S. R. Cloude, "Forest height mapping using space-borne polarimetric SARinterferometry," Geoscience and Remote Sensing Symposium, 2001. IGARSS'01. IEEE 2001 International, vol. 3, 2001.

[14] G. Sun, K. Ranson, D. Kimes, J. Blair, and K. Kovacs, "Forest vertica structure from GLAS: An evaluation using LVIS and SRTM data," Remote Sensing of Environment, vol. 112, pp. 107-117, 2008.

[15] K. Sarabandi and Y. C. Lin, "Simulation of interferometric SAR response for characterizing the scattering phase center statistics of forest canopies," Geoscience and Remote Sensing, IEEE Transactions on, vol. 38, pp. 115-125, 2000.

[16] L. Thirion, E. Colin, and C. Dahon, "Capabilities of a forest coherent scattering model applied to radiometry, interferometry, and polarimetry at P-and L-band," Geoscience and Remote Sensing, IEEE Transactions on, vol. 44, pp. 849-862, 2006.

[17] F. Garestier, P. C. Dubois-Fernandez, and K. P. Papathanassiou, "Pine forest height inversion using single-pass X-band PolInSAR data," Geoscience and Remote Sensing, IEEE Transactions on, vol. 46, pp. 59-68, 2008.

[18] R. N. Treuhaft and P. R. Siqueira, "Vertical structure of vegetated land surfaces from interferometric and polarimetric radar," Radio Science, vol. 35, pp. 141-177, 2000

[19] T. Mette, K. Papathanassiou, I. Hajnsek, H. Pretzsch, and P. Biber, "Applying a common allometric equation to convert forest height from Pol-InSAR data to forest biomass," Geoscience and Remote Sensing Symposium, 2004. IGARSS'04. Proceedings. 2004 IEEE International, vol. 1,2004

[20] E. D. Wallington and I. H. Woodhouse, "Forest height retrieval from commercial X-band SAR products," Geoscience and Remote Sensing, IEEE Transactions on, vol. 44, pp. 863-870, 2006.

[21] I. Woodhouse, E. Wallington, and D. Turner, "Edge effects on tree height retrieval using X-band interferometry," Geoscience and Remote Sensing Letters, IEEE, vol. 3, pp. 344-348, 2006.

[22] I. Izzawati, E. Wallington, and I. Woodhouse, "Forest height retrieval from commercial X-band SAR products," IEEE Transactions on Geoscience and Remote Sensing, vol. 44, 2006.

[23] I. Hajnsek, F. Kugler, S. K. Lee, and K. P. Papathanassiou, "Tropicalforest-parameter estimation by means of Pol-InSAR: The INDREX-II campaign," Geoscience and Remote Sensing, IEEE Transactions on, vol. 47, pp. 481-493, 2009.

[24] H. Skriver, W. Dierking, P. Gudmandsen, T. Le Toan, A. Moreira, K. Papathanassiou, et al., "Applications of synthetic aperture radar polarimetry," in Applications of SAR Polarimetry and Polarimetric Interferometry, 2003, p. 2.

[25] F. Garestier and T. Le Toan, "Forest modeling for height inversion using single-baseline InSAR/Pol-InSAR data," Geoscience and Remote Sensing, IEEE Transactions on, vol. 48, pp. 1528-1539, 2010.

[26] J. B. Blair, D. L. Rabine, and M. A. Hofton, "The Laser Vegetation Imaging Sensor: a medium-altitude, digitisation-only, airborne laser altimeter for mapping vegetation and topography," ISPRS Journal of Photogrammetry and Remote Sensing, vol. 54, pp. 115-122, 1999.

[27] P. Hyde, R. Nelson, D. Kimes, and E. Levine, "Exploring LiDARRaDAR synergy-Predicting aboveground biomass in a southwestern ponderosa pine forest using LiDAR, SAR and InSAR," Remote Sensing of Environment, vol. 106, pp. 28-38, 2007.

[28] R. F. Nelson, P. Hyde, P. Johnson, B. Emessiene, M. L. Imhoff, R. Campbell, et al., "Investigating RaDAR-LiDAR synergy in a North Carolina pine forest," Remote Sensing of Environment, vol. 110, pp. 98108, 2007.

[29] G. Sun, K. J. Ranson, Z. Guo, Z. Zhang, P. Montesano, and D. Kimes, "Forest biomass mapping from lidar and radar synergies," Remote Sensing of Environment, vol. 115, pp. 2906-2916, 2011.

[30] M. Simard, V. H. Rivera-Monroy, J. E. Mancera-Pineda, E. CastañedaMoya, and R. R. Twilley, "A systematic method for 3D mapping of mangrove forests based on Shuttle Radar Topography Mission elevation data, ICEsat/GLAS waveforms and field data: Application to Ciénaga Grande de Santa Marta, Colombia," Remote Sensing of Environment, vol. 112, pp. 2131-2144, 2008.

[31] W. Ni-Meister, D. L. B. Jupp, and R. Dubayah, "Modeling lidar waveforms in heterogeneous and discrete canopies," Geoscience and Remote Sensing, IEEE Transactions on, vol. 39, pp. 1943-1958, 2001.

[32] J. B. Drake, R. O. Dubayah, R. G. Knox, D. B. Clark, and J. Blair, "Sensitivity of large-footprint lidar to canopy structure and biomass in a neotropical rainforest," Remote Sensing of Environment, vol. 81, pp. 378-392, 2002.

[33] M. A. Lefsky, W. Cohen, S. Acker, G. G. Parker, T. Spies, and D. Harding, "Lidar remote sensing of the canopy structure and biophysical properties of Douglas-fir western hemlock forests," Remote Sensing of Environment, vol. 70, pp. 339-361, 1999.

[34] D. Harding, M. Lefsky, G. Parker, and J. Blair, "Laser altimeter canopy height profiles: Methods and validation for closed-canopy, broadleaf forests," Remote Sensing of Environment, vol. 76, pp. 283-297, 2001

[35] R. O. Dubayah and J. B. Drake, "Lidar remote sensing for forestry," Journal of Forestry, vol. 98, pp. 44-46, 2000.

[36] S. R. Cloude, "Polarization coherence tomography," Radio Science, vol. 41, p. RS4017, 2006. 
[37] A. Reigber and A. Moreira, "First demonstration of airborne SAR tomography using multibaseline L-band data," Geoscience and Remote Sensing, IEEE Transactions on, vol. 38, pp. 2142-2152, 2000.

[38] M. Lavalle, M. Simard, and S. Hensley, "A temporal decorrelation model for polarimetric radar interferometers," Geoscience and Remote Sensing, IEEE Transactions on, vol. 50, pp. 2880-2888, 2012.

[39] O. Frey and E. Meier, "3-D time-domain SAR imaging of a forest using airborne multibaseline data at L-and P-bands," Geoscience and Remote Sensing, IEEE Transactions on, vol. 49, pp. 3660-3664, 2011.

[40] O. Frey and E. Meier, "Analyzing tomographic SAR data of a forest with respect to frequency, polarization, and focusing technique," Geoscience and Remote Sensing, IEEE Transactions on, vol. 49, pp. 3648-3659, 2011.

[41] Y. Huang, L. Ferro-Famil, and A. Reigber, "Under-foliage object imaging using SAR tomography and polarimetric spectral estimators," Geoscience and Remote Sensing, IEEE Transactions on, vol. 50, pp. 2213-2225, 2012.

[42] S. Tebaldini and F. Rocca, "Multibaseline polarimetric SAR tomography of a boreal forest at P-and L-bands," Geoscience and Remote Sensing, IEEE Transactions on, vol. 50, pp. 232-246, 2012.

[43] O. Ponce, P. Prats-Iraola, M. Pinheiro, M. Rodriguez-Cassola, R. Scheiber, A. Reigber, et al., "Fully polarimetric high-resolution 3-D imaging with circular SAR at L-band," Geoscience and Remote Sensing, IEEE Transactions on, vol. 52, pp. 3074-3090, 2014.

[44] S. Hensley, K. Wheeler, G. Sadowy, C. Jones, S. Shaffer, H. Zebker, et al., "The UAVSAR instrument: Description and first results," in Radar Conference, 2008. RADAR'08. IEEE, 2008, pp. 1-6.

[45] W. Huang, G. Sun, R. Dubayah, Z. Zhang, and W. Ni, "Mapping forest above-ground biomass and its changes from LVIS waveform data," in Geoscience and Remote Sensing Symposium (IGARSS), 2012 IEEE International, 2012, pp. 6561-6564.

[46] P. Hyde, R. Dubayah, B. Peterson, J. Blair, M. Hofton, C. Hunsaker, et al., "Mapping forest structure for wildlife habitat analysis using waveform lidar: Validation of montane ecosystems," Remote Sensing of Environment, vol. 96, pp. 427-437, 2005.

[47] A. Fu, G. Sun, and Z. Guo, "Estimating forest biomass with GLAS samples and MODIS imagery in Northeastern China," in Sixth International Symposium on Multispectral Image Processing and Pattern Recognition, 2009, pp. 749844-749844-8.

[48] Z. Wang, C. B. Schaaf, P. Lewis, Y. Knyazikhin, M. A. Schull, A. H. Strahler, et al., "Retrieval of canopy height using moderate-resolution imaging spectroradiometer (MODIS) data," Remote Sensing of Environment, vol. 115, pp. 1595-1601, 2011.

[49] M. Simard, S. Hensley, M. Lavalle, R. Dubayah, N. Pinto, and M. Hofton, "An Empirical Assessment of Temporal Decorrelation Using the Uninhabited Aerial Vehicle Synthetic Aperture Radar over Forested Landscapes," Remote Sensing, vol. 4, pp. 975-986, 2012.

[50] N. Pinto, M. Simard, and R. Dubayah, "Using InSAR coherence to map stand age in a boreal forest," Remote Sensing, vol. 5, pp. 42-56, 2012.

[51] M. Hofton, J. B. Minster, and J. B. Blair, "Decomposition of laser altimeter waveforms," Geoscience and Remote Sensing, IEEE Transactions on, vol. 38, pp. 1989-1996, 2000.

[52] R. Ahmed, P. Siqueira, and S. Hensley, "A study of forest biomass estimates from lidar in the northern temperate forests of New England," Remote Sensing of Environment, vol. 130, pp. 121-135, 2013.

[53] D. Y. Hollinger, S. Ollinger, A. Richardson, T. Meyers, D. Dail, M. Martin, et al., "Albedo estimates for land surface models and support for a new paradigm based on foliage nitrogen concentration," Global Change Biology, vol. 16, pp. 696-710, 2010.

[54] D. Post, A. Fimbres, A. Matthias, E. Sano, L. Accioly, A. Batchily, et al., "Predicting soil albedo from soil color and spectral reflectance data," Soil Science Society of America Journal, vol. 64, pp. 1027-1034, 2000.

[55] R. H. MacArthur and H. S. Horn, "Foliage profile by vertical measurements," Ecology, pp. 802-804, 1969.

[56] M. A. Lefsky, D. Harding, W. Cohen, G. Parker, and H. Shugart, "Surface lidar remote sensing of basal area and biomass in deciduous forests of eastern Maryland, USA," Remote Sensing of Environment, vol. 67, pp. 83-98, 1999.

[57] J. T. Pulliainen, L. Kurvonen, and M. T. Hallikainen, "Multitemporal behavior of L-and C-band SAR observations of boreal forests," Geoscience and Remote Sensing, IEEE Transactions on, vol. 37, pp. 927-937, 1999.

[58] E. P. W. Attema and F. T. Ulaby, "Vegetation modeled as a water cloud," Radio Science, vol. 13, pp. 357-364, 1978
[59] S. Cloude, M. Brolly, and I. Woodhouse, "A study of forest vertical structure estimation using coherence tomography coupled to a macroecological scattering model," 2009, pp. IV-717-IV-720.

[60] A. T. Caicoya, F. Kugler, K. Papathanassiou, P. Biber, and H. Pretzsch, "Biomass estimation as a function of vertical forest structure and forest height-Potential and limitations for Radar Remote Sensing," in Synthetic Aperture Radar (EUSAR), 2010 8th European Conference on, 2010, pp. 1-4.

[61] F. T. Ulaby, A. Tavakoli, and B. A. Thomas, "Microwave propagation constant for a vegetation canopy with vertical stalks," Geoscience and Remote Sensing, IEEE Transactions on, pp. 714-725, 1987.

[62] M. A. Lefsky, D. J. Harding, M. Keller, W. B. Cohen, C. C. Carabajal, F. Del Bom Espirito-Santo, et al., "Estimates of forest canopy height and aboveground biomass using ICESat," Geophysical Research Letters, vol. 32, 2005

[63] H. Tang, R. Dubayah, A. Swatantran, M. Hofton, S. Sheldon, D. B. Clark, et al., "Retrieval of vertical LAI profiles over tropical rain forests using waveform lidar at La Selva, Costa Rica," Remote Sensing of Environment, vol. 124, pp. 242-250, 2012/06/14/14:55:10 2012.

[64] Y. Kankaku, Y. Osawa, S. Suzuki, and T. Watanabe, "The overview of the L-band SAR onboard ALOS-2," in Proceedings of Progress in Electromagnetics Research Symposium, 2009.

[65] R. Torres, P. Snoeij, D. Geudtner, D. Bibby, M. Davidson, E. Attema, et al., "GMES Sentinel-1 mission," Remote Sensing of Environment, vol. 120, pp. 9-24, 2012.

[66] T. Le Toan, S. Quegan, M. Davidson, H. Balzter, P. Paillou, K. Papathanassiou, et al., "The BIOMASS mission: Mapping global forest biomass to better understand the terrestrial carbon cycle," Remote sensing of environment, vol. 115, pp. 2850-2860, 2011.

[67] O. Alvarez-Salazar, S. Hatch, J. Rocca, P. Rosen, S. Shaffer, Y. Shen, et al., "Mission design for NISAR repeat-pass Interferometric SAR," in SPIE Remote Sensing, 2014, pp. 92410C-92410C-10. 
Matthew Brolly was born in Louth, UK in 1981. He received the B.S. degree

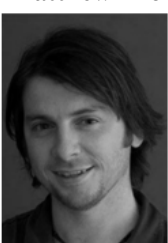
in Geophysics from the University of Edinburgh, UK in 2004 and the M.S. degree in Archaeological Geophysics from the University of Bradford, UK in 2005. Following two years working in the commercial geophysics sector he returned to the University of Edinburgh, UK on a NERC scholarship to receive his $\mathrm{PhD}$ degree in Radar Remote Sensing (2011). From 2011 to 2013 he held a Research Associate position at the University of Maryland, USA before becoming a Lecturer in Geography/Environmental Science at the University of Brighton, UK in 2013. His main research interests lie in active remote sensing, modeling and sensor fusion particularly in the field of environmental science.

Marc Simard is a Senior Scientist at the Jet Propulsion Laboratory's Radar

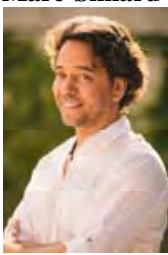

Science \& Engineering Section.

He obtained his Ph.D. in the Department of Geomatics of the Université Laval in 1998. Since then, he has been at JPL, working on several science projects and flight missions (AIRSAR, GeoSAR, UAVSAR, WSOA, SWOT and NISAR). His expertise is in algorithm development of products for ecological science applications based on active remote sensing. He is particularly interested in the fusion of large and small footprint lidars, synthetic aperture radar (SAR) and interferometric SAR to produce 3D renditions of volumes and surfaces such as vegetation canopy and topography over large geographical areas.

Hao Tang received the B.S. degree in Geographical Information System (GIS) from Nanjing University, China in 2009 and the Ph.D. degree in Geography from University of Maryland, College Park in 2015. From 2009 to 2015, he was a Research Assistant at the Department of Geographical Sciences, University of Maryland where he continued his study as a Post-doctoral Research Associate for the Global Ecosystem Dynamics Investigation (GEDI) lidar mission. His research interests primarily focus on characterizing $3 \mathrm{D}$ dynamics of terrestrial ecosystems using various lidar remote sensing platforms. He was also a NASA Earth and Space Science fellow (NESSF) from $2012 ~ 2015$.

Ralph Dubayah received the B.A. degree from the University of California,

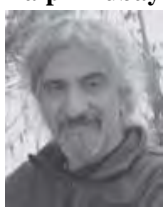
Berkeley, CA, USA, in 1982 and the M.A. and Ph.D. degrees from the University of California, Santa Barbara, CA, in 1985 and 1990, respectively. He is a Professor of geographical sciences with the University of Maryland, College Park, MD, USA. His main areas of interest are ecosystem characterization for carbon modeling, habitat and biodiversity studies, land surface energy and water balance modeling, spatial analysis, and remote sensing science

Justin P. Fisk received the B.A. degree in environmental studies from the

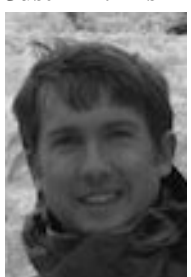
University of Colorado, Boulder in 1998, the M.C.S. degree in computer science from Colorado State University, Fort Collins, in 2004, and the Ph.D. in Earth and environmental science from the University of New Hampshire, Durham in 2015. From 1999 to 2006 he worked in software engineering and high-performance computing in industry. He held research positions at the University of New Hampshire and the University of Maryland between 2009 and 2015. He is currently a senior research scientist at Applied Geosolutions in Newmarket, NH. His research centers on connecting remote sensing with mechanistic ecosystem models, with a focus on ecosystem-atmosphere interactions in coupled human and natural systems. 


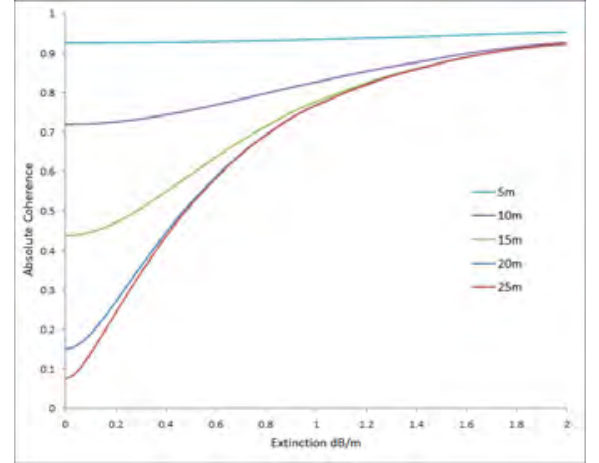

Fig 1. Absolute coherence variations calculated using RVoG model for increasing extinctions at 5 constant forest heights.

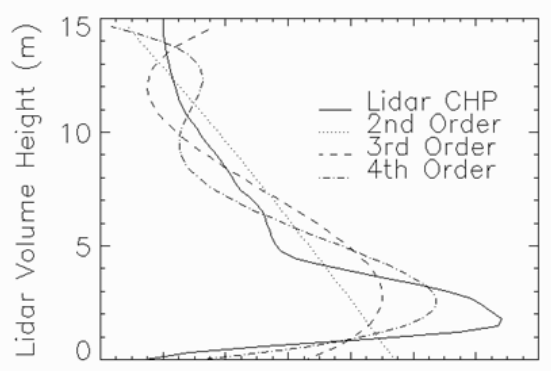

$\begin{array}{llllllll}-0.2 & 0.0 & 0.2 & 0.4 & 0.6 & 0.8 & 1.0 & 1.2\end{array}$ Cumulative Return Intensity Derivative

Fig 2. (Right) Normalised CHP with fitted Legendre polynomial solutions from $2^{\text {nd }}$ to $4^{\text {th }}$ order. All data is shown with respect to volume height as estimated by lidar waveforms. CHP values are calculated through normalising the canopy contribution by the total area beneath the curve thus allowing extinction estimates and variations to be relative to both the canopy and ground contributions. Greater variation in the profile is apparent when one or more of the following are exhibited; greater height, greater extinction, or greater offset variation from 0 at canopy max height. The cCHP can be included in the comparison as it is the integral with respect to height of the CHP.

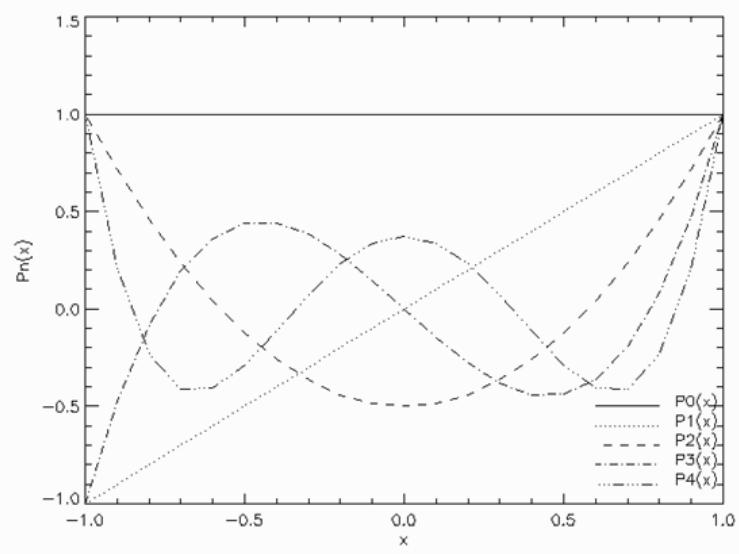

Fig 3. Visual representation of the first 5 Legendre polynomials as a function of $x$.

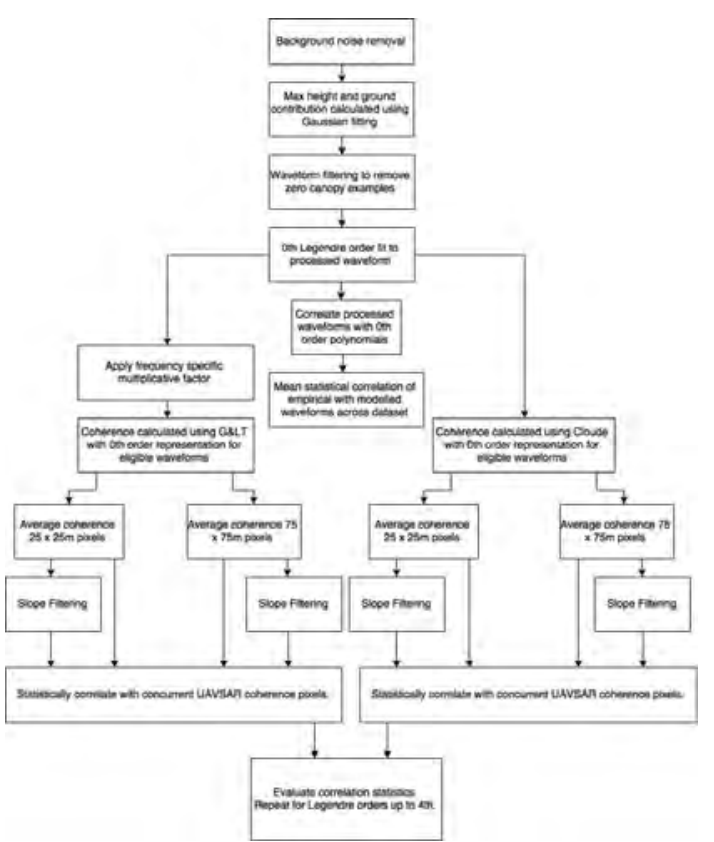

Figure 4. Processing the Lidar waveform to model radar interferometric coherence. Work flow outlining the process of modelling waveforms using Legendre polynomials and coherence estimation. The process is repeated for all orders up to $4^{\text {th }}$ order to provide complete analysis of increasing the complexity of modelled waveforms.

Table 1. Correlation statistics of Legendre polynomials with CHPs derived from lidar waveform data collected within the study area of radar and lidar data overlap.

\begin{tabular}{|l|r|r|}
\hline & Mean $\mathbf{r}^{2}$ & St. Dev $\mathbf{r}^{2}$ \\
\hline \multicolumn{1}{l|}{ CHP } & & \\
\hline $1^{\text {st }}$ Order & 0.44 & 0.20 \\
\hline $2^{\text {nd }}$ Order & 0.63 & 0.14 \\
\hline $3^{\text {rd }}$ Order & 0.77 & 0.17 \\
\hline $4^{\text {th }}$ Order & 0.88 & 0.12 \\
\cline { 1 - 3 } & &
\end{tabular}




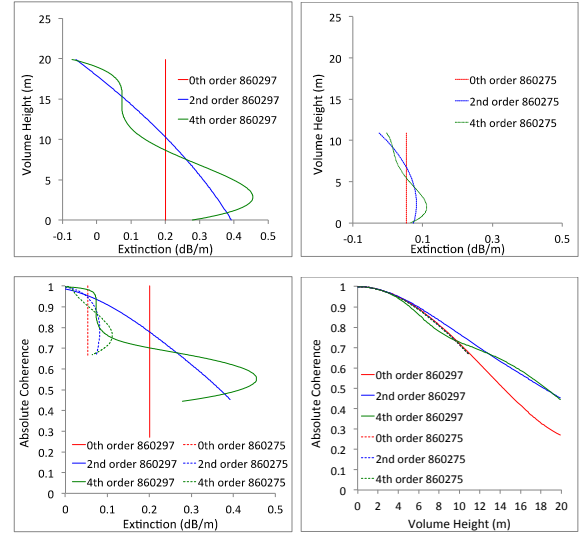

Fig 5. G\&LT model Coherence estimations resulting from extinction with canopy depth. (Top Left) Extinction variation shown up to $4^{\text {th }}$ Legendre order for waveform number 860297 as a function of volume height, (Top Right) Extinction variation shown up to $4^{\text {th }}$ order for waveform number 860275 as a function of volume height. (Bottom Left) Absolute coherence as a function of extinction up to $4^{\text {th }}$ order for both waveforms. (Bottom Right) Absolute coherence as a function of volume height up to the $4^{\text {th }}$ order for both waveforms.

Table 2. $r^{2}$ values associated with correlation between lidar estimated and UAVSAR interferometric coherence using the Cloude and Garestier \& Le Toan models. Representative data shown for hh, vv, and hv polarisations up to $4^{\text {th }}$ Legendre order. Data presented for slope angle filtering $\left(<30^{\circ}\right)$. All correlations shown for $\mathrm{p}<0.05$.

\begin{tabular}{|c|c|c|c|c|c|c|}
\hline \multirow[b]{2}{*}{ Legendre Orde } & \multicolumn{2}{|l|}{ hh } & \multicolumn{2}{|l|}{$\mathrm{vv}$} & \multicolumn{2}{|l|}{ hv } \\
\hline & & & & & & \\
\hline G\&LT & $r^{2}$ & $\begin{array}{c}(75 \mathrm{~m} \\
\mathrm{x} 75 \mathrm{~m}) \\
\mathrm{r}^{2}\end{array}$ & $r^{2}$ & $\begin{array}{c}(75 \mathrm{~m} \\
\times 75 \mathrm{~m}) \\
\mathrm{r}^{2}\end{array}$ & $r^{2}$ & $\begin{array}{c}(75 \mathrm{~m} \\
\times 75 \mathrm{~m}) \\
\mathrm{r}^{2}\end{array}$ \\
\hline $0^{\text {th }}$ & 0.44 & 0.73 & 0.42 & 0.71 & 0.48 & 0.74 \\
\hline 1st & 0.46 & 0.73 & 0.44 & 0.71 & 0.50 & 0.74 \\
\hline 2nd & 0.46 & 0.73 & 0.44 & 0.71 & 0.50 & 0.74 \\
\hline 3rd & 0.46 & 0.73 & 0.45 & 0.71 & 0.51 & 0.74 \\
\hline 4th & 0.43 & 0.72 & 0.41 & 0.70 & 0.47 & 0.73 \\
\hline \multicolumn{7}{|l|}{ Cloude } \\
\hline 0th & 0.43 & 0.72 & 0.40 & 0.70 & 0.46 & 0.73 \\
\hline 1 st & 0.43 & 0.72 & 0.40 & 0.70 & 0.46 & 0.73 \\
\hline 2nd & 0.43 & 0.72 & 0.40 & 0.70 & 0.47 & 0.73 \\
\hline $3 r d$ & 0.43 & 0.73 & 0.40 & 0.70 & 0.47 & 0.74 \\
\hline 4th & 0.45 & 0.74 & 0.43 & 0.71 & 0.49 & 0.75 \\
\hline \multicolumn{7}{|c|}{ Constant Ext. (RVoG) } \\
\hline $0.001 \mathrm{~dB} / \mathrm{m}$ & 0.43 & 0.73 & 0.40 & 0.70 & 0.47 & 0.74 \\
\hline $0.15 \mathrm{~dB} / \mathrm{m}$ & 0.44 & 0.74 & 0.41 & 0.71 & 0.48 & 0.75 \\
\hline $0.5 \mathrm{~dB} / \mathrm{m}$ & 0.44 & 0.75 & 0.40 & 0.71 & 0.48 & 0.76 \\
\hline $1 \mathrm{~dB} / \mathrm{m}$ & 0.21 & 0.63 & 0.20 & 0.60 & 0.23 & 0.64 \\
\hline
\end{tabular}

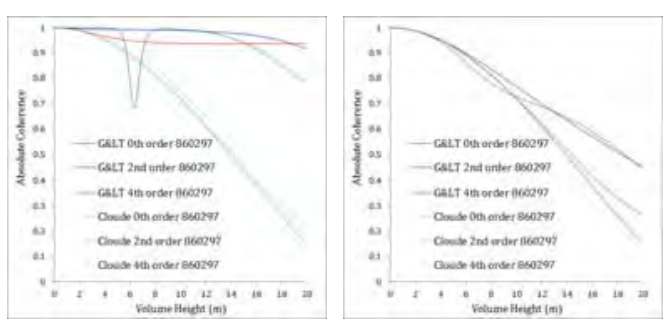

Fig 6. Absolute coherence plotted against volume height for a single waveform (\#. 860297) representing a forest component of $20 \mathrm{~m}$ height. Representative data is shown using both the G\&LT and Cloude models ((left) without multiplicative factor applied, (right) with multiplicative factor applied). $0^{\text {th }}$ order representations of the G\&LT modelled coherence data, show greatest similarity to those of the Cloude model.

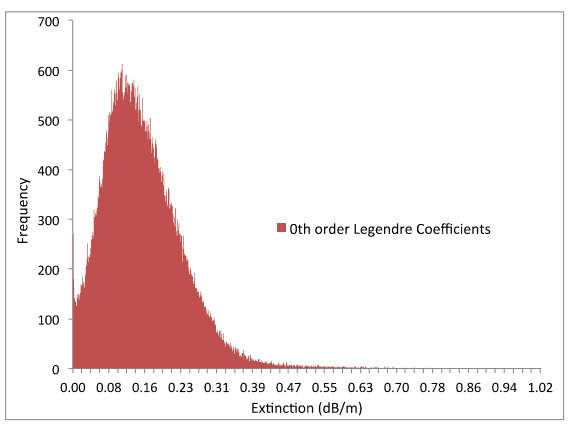

Fig 7. Frequency of occurrence of extinction values associated with lidar waveform processing using CHPs following application of multiplicative factor. Extinction values are estimated using $0^{\text {th }}$ order Legendre polynomial representations of waveforms. Prior to multiplicative factor mean value $1.67 \mathrm{~dB} / \mathrm{m}$. Multiplicative factor applied is $0.09((0.15 \mathrm{~dB} / \mathrm{m}) /(1.67 \mathrm{~dB} / \mathrm{m}))$ 

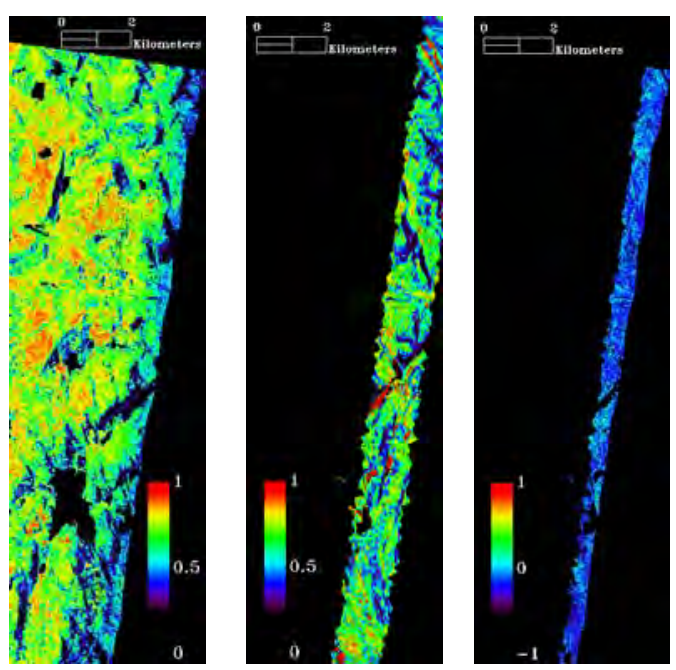

Fig 8. (Left) Interferometric coherence from UAVSAR HV L-Band data displayed on a scale 0 to 1 . (Centre) Estimated interferometric coherence using lidar data with the G\&LT model, incorporating $2^{\text {nd }}$ order Legendre polynomial representations of the CHP profiles (includes multiplicative factor). (Right) Area of overlap between lidar and radar data highlighted through subtraction of lidar estimates from radar values. Data shows the difference between datasets using a scale of -1 to 1 . Other Legendre orders present similar visual data with differences between orders difficult to visually determine.

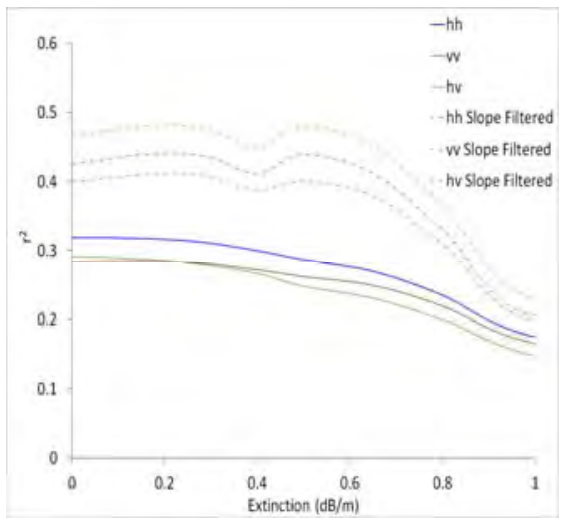

Fig 9. $r^{2}$ estimates across the test site made using RVoG modelled interferometric coherence estimation (eq 7, using constant mean extinction) compared with UAVSAR observed coherence. Data is associated with $25 \mathrm{~m}$ resolution for entire overlapping area (Solid lines) and for the same dataset excluding slopes exceeding $30^{\circ}$ (Dotted lines). Data can be used for comparison with $\mathrm{r}^{2}$ values presented using the G\&LT and Cloude models and Legendre interpretations of extinction. Heights used in all calculations derived from waveforms.
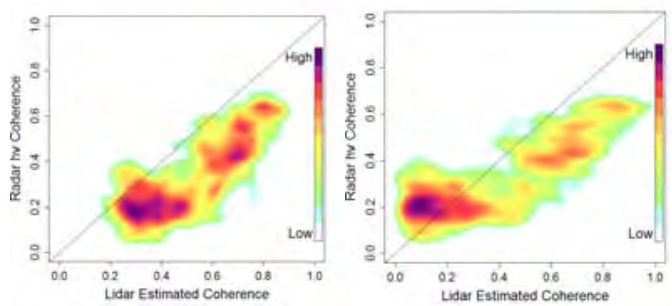

Fig 10. Scattering density plots of UAVSAR hv interferometric coherence against LVIS lidar estimated coherence using (Left) CHP G\&LT model $3^{\text {rd }}$ order legendre with slope filtering applied $\left(<30^{\circ}\right)\left(\mathrm{r}^{2}\right.$ of 0.51$)$ (Right) CHP Cloude model $4^{\text {th }}$ order legendre with slopes $<30^{\circ}\left(r^{2}\right.$ of 0.49$)$.. Warm colours represent greater concentration of data. 